(1)

CrossMark

\title{
Pulmonary xenon-129 MRI: new opportunities to unravel enigmas in respiratory medicine
}

\author{
Rachel L. Eddy ${ }^{1,2}$ and Grace Parraga ${ }^{1,2,3}$ \\ Affiliations: ${ }^{1}$ Robarts Research Institute, London, ON, Canada. ${ }^{2}$ Dept of Medical Biophysics, Western \\ University, London, ON, Canada. ${ }^{3}$ Division of Respirology, Dept of Medicine, Western University, London, ON, \\ Canada.
}

Correspondence: Grace Parraga, Robarts Research Institute, 1151 Richmond St N, London, ON, Canada, N6A 5B7. E-mail: gparragadrobarts.ca

@ERSpublications

${ }^{129} \mathrm{Xe}$ MRI provides rapid, sensitive, non-invasive, high spatial resolution and simultaneous measurements of pulmonary ventilation, tissue microstructure and gas exchange, and is poised for routine clinical assessments in patients with chronic lung disease http://bit.ly/2PKkFcU

Cite this article as: Eddy RL, Parraga G. Pulmonary xenon-129 MRI: new opportunities to unravel enigmas in respiratory medicine. Eur Respir J 2020; 55: 1901987 [https://doi.org/10.1183/13993003.019872019].

\section{Introduction}

Computed tomography (CT) of the chest is the imaging modality of choice for the non-invasive, quantitative evaluation of chronic lung diseases, because thoracic CT protocols are nearly ubiquitously available and provide rapid, high-resolution images of the airway, and parenchymal structure and anatomy. Pulmonary magnetic resonance imaging (MRI) has not been used clinically, mainly because of complexity (pulmonary MRI signal decays rapidly), cost and because conventional MRI is dependent on proton density (hydrogen atoms in tissue), which is exceptionally low (around $0.1 \mathrm{~g} \cdot \mathrm{cm}^{-3}$ ) in the healthy lung, because it mainly comprises air rather than tissue or being water-filled $[1,2]$. Using conventional MRI methods, the lungs mainly appear as dark, signal-deficient voids and the pulmonary MRI signal is further degraded because of the millions of lung air-tissue interfaces that cause local magnetic field distortions, and respiratory and cardiac motion. While anatomical proton MRI of the lung is now developing rapidly to overcome these technical limitations, it still does not provide information beyond that of a low-dose CT, so there is a drive towards functional MRI. One of these approaches involves the use of inhaled gas contrast agents, primarily hyperpolarised (or magnetised) helium-3 $\left({ }^{3} \mathrm{He}\right)$ and xenon-129 $\left({ }^{129} \mathrm{Xe}\right)$, both of which provide a way to rapidly $(<10 \mathrm{~s})$ and directly visualise inhaled gas distribution with high spatial resolution $(\sim 3 \mathrm{~mm} \mathrm{x}, \mathrm{y}$ and $\mathrm{z}$ planes). Pulmonary functional MRI is also possible using inhaled fluorinated gas [3], oxygen-enhanced techniques [4] and free-breathing proton methods [5, 6]; however, hyperpolarised inhaled gas MRI has been the most widely used and described. Because inhaled contrast gases have different resonant frequencies than hydrogen protons as used for conventional MRI, these methods inherently have no background signal and excellent contrast.

While inhalation of hyperpolarised gases to measure pulmonary function was originally discovered using ${ }^{129} \mathrm{Xe}$ [7], the field was dominated by ${ }^{3} \mathrm{He}$ MRI until recently, because of superior ${ }^{3} \mathrm{He}$ MRI image quality with low volume $(<500 \mathrm{~mL})$ inhaled doses. However, the limited worldwide supply of ${ }^{3} \mathrm{He}[8]$ has driven the field back to ${ }^{129} \mathrm{Xe}$ gas, because it is naturally abundant and turnkey polarisation technologies have 
significantly advanced [9-11]. ${ }^{129} \mathrm{Xe}$ also has modest solubility in biological tissues and resonates at different frequencies when dissolved in different tissues, so it provides novel alveolar tissue and capillary blood information. Here, we provide an overview of the key concepts and methods for ${ }^{129} \mathrm{Xe}$ MRI, and discuss the current state-of-the-art and how ${ }^{129}$ Xe MRI may be applied in the future.

\section{How does ${ }^{129}$ Xe MRI work?}

${ }^{129} \mathrm{Xe} \mathrm{MRI}$ is dependent on equipment that polarises ${ }^{129} \mathrm{Xe}$ atoms, to effectively increase the nuclear polarisation by approximately 100000 times. Commercial (Polarean Inc., Durham, NC, USA; Xemed LLC, Durham, NH, USA) and custom-built $[10,11]$ polariser systems operate via spin-exchange optical pumping [12] whereby circularly polarised light bombards a glass cell housing rubidium and ${ }^{129} \mathrm{Xe}$. The circularly polarised light is absorbed by rubidium and subsequent collisions between polarised rubidium and ${ }^{129} \mathrm{Xe}$ transfer angular momentum to ${ }^{129} \mathrm{Xe}$ and increase its nuclear-spin polarisation. Typically, isotopically enriched ${ }^{129} \mathrm{Xe}\left(\sim 86 \%\right.$ by volume) is used to further increase the fraction of ${ }^{129} \mathrm{Xe}$ atoms that are polarised in a given volume. ${ }^{129} \mathrm{Xe}$ flows through the glass cell at a constant rate and the hyperpolarised ${ }^{129} \mathrm{Xe}$ is frozen as it leaves the cell and remains frozen until the desired amount is accumulated, upon which it is thawed and dispensed into a bag for patient inhalation. The ${ }^{129} \mathrm{Xe}$ flow rate through the cell can be adjusted to accumulate the desired ${ }^{129} \mathrm{Xe}$ dose within a desired time frame. ${ }^{129} \mathrm{Xe}$ doses vary from $250 \mathrm{~mL}$ up to $1.0 \mathrm{~L}$ [13], and doses are typically diluted up to $1.0 \mathrm{~L}$ using medical-grade nitrogen $\left(\mathrm{N}_{2}\right)$ or helium-4. ${ }^{129} \mathrm{Xe}$ hyperpolariser equipment is typically located in a small room adjacent to the MRI suite, within close proximity to deliver the polarised gas to the patient as quickly as possible.

${ }^{129} \mathrm{Xe}$ MRI is feasible on both $1.5 \mathrm{~T}$ and $3.0 \mathrm{~T}$ field strength scanners. To acquire the signal, however, a radiofrequency coil is fitted around the patient's chest and this is specifically tuned to the resonance frequency of ${ }^{129} \mathrm{Xe}$. There are a number of different ${ }^{129} \mathrm{Xe}$ coils; however, as shown in figure 1, the most common are quadrature rigid birdcage or flexible vests (Clinical MR Solutions, Brookfield, WI, USA; RAPID Biomedical, Rimpar, Germany). Rigid birdcage coils provide more homogeneous MRI signal throughout the lungs (i.e. no artefactual differences in MRI signal that are unrelated to anatomy) and increased signal-to-noise, but they have patient size limitations; flexible vest coils can accommodate a larger range of patient sizes but may require additional image corrections after MRI acquisition [14] to account for signal differences caused by the way the patient and coil interact. Additional receive array-coils may be placed on the patient's chest inside the rigid or flexible design coils, and this improves signal-to-noise ratios and accelerates acquisition times, which enables very short breath-hold scans $(\sim 5 \mathrm{~s})$. Figure 1a shows ${ }^{129}$ Xe MRI equipment including hyperpolariser, gas delivery bag and radiofrequency coils, and patient set-up in MRI scanner.

${ }^{129} \mathrm{Xe}$ MRI is primarily performed under breath-hold conditions, though dynamic multi-breath protocols have also been applied [15]. While laying supine, patients are coached to inhale the gas mixture from passive end expiration or functional residual capacity and hold their breath while the image is acquired, which may take from 5 to $16 \mathrm{~s}$. Pulmonary ${ }^{129} \mathrm{Xe}$ MRI acquisitions may include static ventilation, diffusion-weighted or dissolved-phase methods. Static ventilation images are the most commonly reported ${ }^{129} \mathrm{Xe}$ MRI acquisition and this provides regional maps of pulmonary gas distribution. A conventional proton $\left({ }^{1} \mathrm{H}\right)$ image is also typically acquired in the same scanning session and the same lung volume so that the anatomical and functional images may be co-registered to distinguish the edges of the thoracic cavity during image analysis and segmentation. Figure $1 \mathrm{~b}$ shows a ${ }^{129} \mathrm{Xe}$ gas static ventilation coronal image (cyan) and corresponding anatomical proton image (greyscale), and the two co-registered demonstrating a regional ventilation map. A corresponding CT slice is also shown in figure 1 as well as the three-dimensional segmented airway tree (yellow), which may be co-registered to the ventilation map to demonstrate structure-function relationships.

Diffusion-weighted ${ }^{129}$ Xe MRI methods may also be employed and these estimate the self-diffusion of ${ }^{129} \mathrm{Xe}$ gas within the terminal airways and their restriction within the airspaces on a voxel-wise basis [16], which provides an excellent surrogate measurement of alveolar and terminal bronchiole enlargement.

${ }^{129} \mathrm{Xe}$ has a large, loosely bound electron cloud, making it sensitive to its surroundings and soluble in biological tissue; once dissolved in biological tissues, ${ }^{129} \mathrm{Xe}$ exhibits a different resonance frequency. Thus, ${ }^{129} \mathrm{Xe}$ MRI can also provide in vivo measurements of pulmonary gas exchange $[17,18]$. The so-called dissolved-state refers to ${ }^{129} \mathrm{Xe}$ dissolved in the alveolar membrane and in red blood cells. ${ }^{129} \mathrm{Xe}$ that has diffused into biological tissues experiences a different chemical environment and this is reflected via a shift in the ${ }^{129} \mathrm{Xe}$ resonance frequency (chemical shift) from gaseous ${ }^{129} \mathrm{Xe}$, as shown in figure $1 \mathrm{~b}$. In the same manner, there is also a difference in the resonance frequency between ${ }^{129} \mathrm{Xe}$ in the tissue and ${ }^{129} \mathrm{Xe}$ in the blood, which enables simultaneous, independent imaging of these three states or compartments: 1) gas, 2) tissue barrier plus plasma, and 3) red blood cell. The gas state reflects the inhaled gas and has the largest measurable signal. ${ }^{129} \mathrm{Xe}$ dissolved in the tissue barrier and blood plasma have indistinguishable 
a)

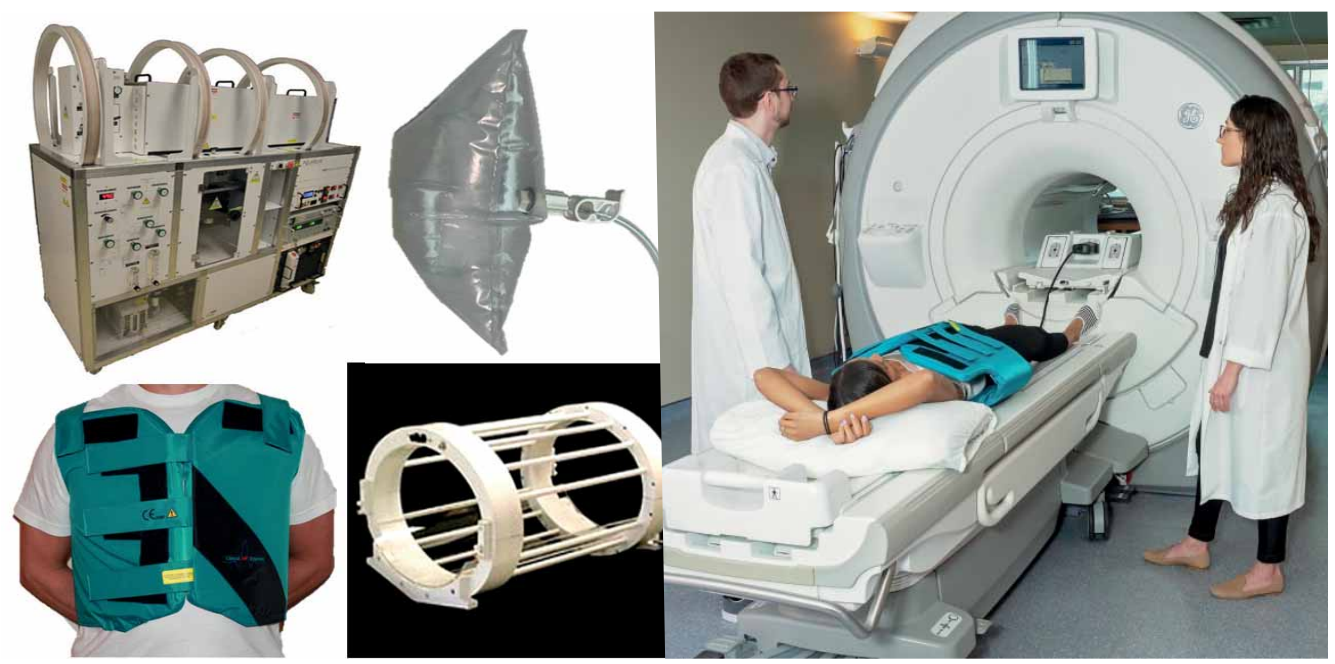

Co-registered
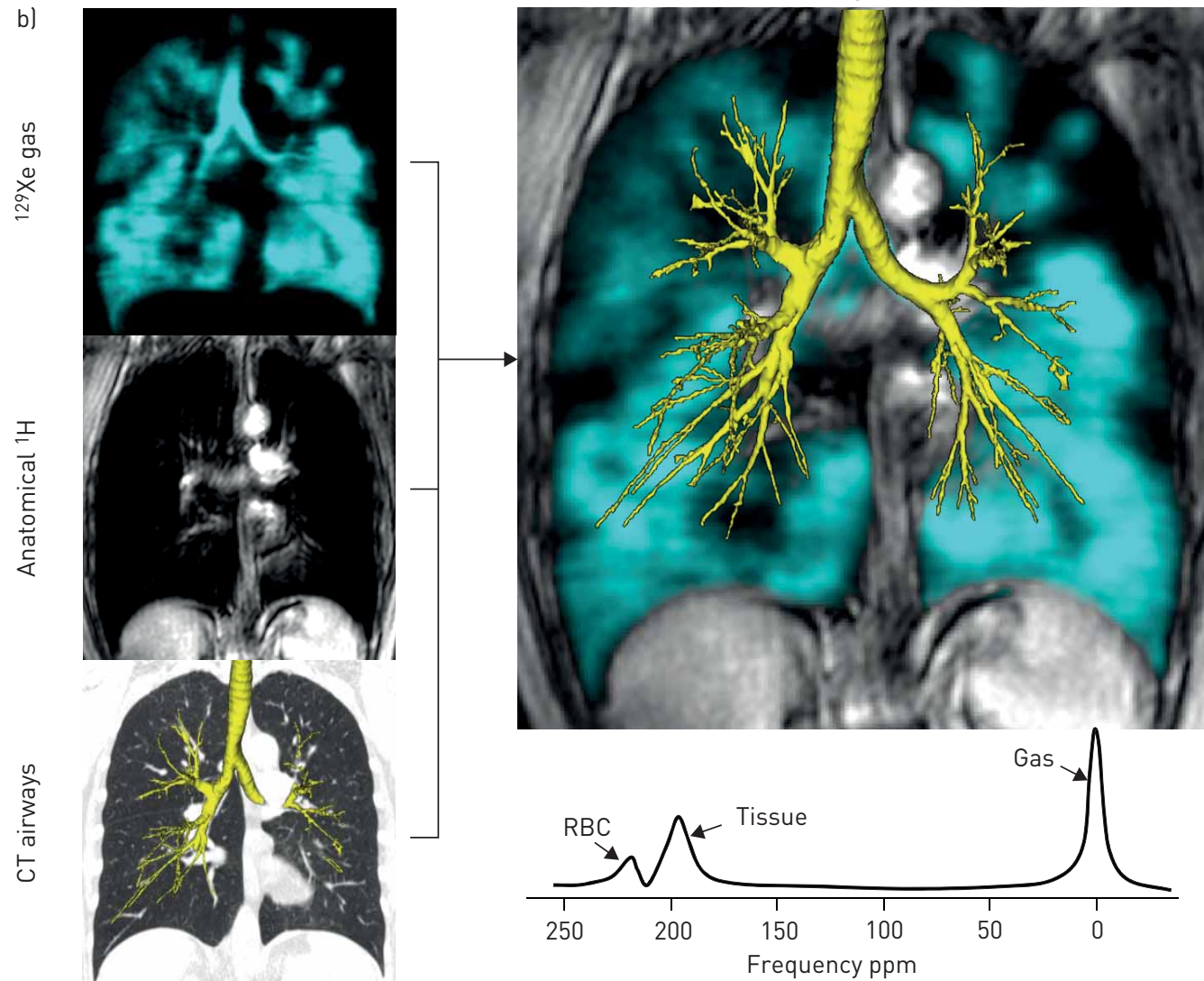

FIGURE 1 Xenon-129 magnetic resonance imaging (MRI) infrastructure requirements and image acquisition. a) ${ }^{129} \mathrm{Xe}$ hyperpolariser, gas delivery bag, radiofrequency coils (flexible vest and rigid birdcage), and patient set-up for MRI. b) ${ }^{129} \mathrm{Xe}$ gas (cyan), anatomical proton $\left({ }^{1} \mathrm{H}\right.$ ), and computed tomography (CT) airway (yellow) images co-registered, with ${ }^{129} \mathrm{Xe}$ gas, tissue plus plasma and red blood cell (RBC) spectrum.

chemical shifts and combine for the second largest measurable signal with a $\sim 197 \mathrm{ppm}$ shift from gaseous ${ }^{129} \mathrm{Xe}$ [19]. ${ }^{129} \mathrm{Xe}$ dissolved in red blood cells exhibits an additional $\sim 20 \mathrm{ppm}$ shift beyond the tissue-plasma signal and makes up the smallest, measurable ${ }^{129} \mathrm{Xe}$ signal [19]; moreover, the red blood cell signal is oxygen-dependent and may undergo another measurable $\sim 5 \mathrm{ppm}$ shift based on blood oxygenation [20]. Tuning the scanner allows acquisition of images of all three compartments, and acquisition protocols have been developed to simultaneously acquire quantitative images from all three compartments within a single breath-hold [18]. 


\section{Current state-of-the-art}

${ }^{129}$ Xe MRI methods have an excellent safety profile in patients with respiratory disease [21, 22], including asthma [23-25], COPD [16, 26-29], cystic fibrosis [30-32], pulmonary vascular disease [33], idiopathic pulmonary fibrosis [34, 35], lung cancer [36] and lymphangioleiomyomatosis [37].

In healthy volunteers, ${ }^{29} \mathrm{Xe}$ gas distribution is typically highly homogeneous and gas signal fills the entire lung whereas in patients with lung disease such as in figure 1, focal MRI signal voids or ventilation defects and patchy gas distribution are often observed. Ventilation defects are commonly quantified as the ventilation defect percent (VDP) [38]: the volume of ventilation defects normalised to the thoracic cavity volume. In a similar manner, using diffusion-weighted MRI, the apparent diffusion coefficient (ADC), a measure of airspace enlargement, may be estimated. ${ }^{129} \mathrm{Xe}$ ADC is low in healthy participants and increases with increasing airspace size and the extent of emphysema [16, 26]. Diffusion-weighted MRI may also be used to generate morphological airspace measurements [39, 40], for example mean linear intercept analogous to histology. Normalised biomarkers of the ratio of ${ }^{129} \mathrm{Xe}$ dissolved in the tissue barrier and red blood cells [18] have been shown to be particularly relevant in pulmonary fibrosis. A unique feature of ${ }^{129} \mathrm{Xe} \mathrm{MRI}$ is that it provides a way to regionally quantify gas exchange $[17,41]$ in regions of the lung that are ventilated and models have been developed to extract subcomponents of gas exchange and structural measurements [42-44].

At the current time, ${ }^{129} \mathrm{Xe}$ MRI is approved for clinical use in the UK and clinical approval is pending in the USA.

\section{How is ${ }^{129} \mathrm{Xe}$ MRI likely to be used in the future?}

The infrastructure needed to enable ${ }^{129} \mathrm{Xe}$ MRI is available in $\sim 12$ respiratory imaging sites worldwide using a wide variety of different scanners, polarisers and coils. Whilst technical developments will enable faster ${ }^{129} \mathrm{Xe}$ polarisation times and larger volumes of polarised gas, multicentre clinical trials are now poised to demonstrate the inter- and intra-site reproducibility of ${ }^{129} \mathrm{Xe}$ MRI biomarkers so that clinical trials of new treatments and interventions using MRI may be powered. A comprehensive ${ }^{129} \mathrm{Xe}$ examination (including localiser scan, anatomical scan, ventilation, diffusion-weighted, perfusion and gas exchange scans) in a patient may be easily performed within $15 \mathrm{~min}$, often with patients inside the MRI bore for approximately $5 \mathrm{~min}$ and this is certainly compatible with current clinical imaging workflows.

Highly sensitive ${ }^{129} \mathrm{Xe}$ MRI VDP promises therapy studies utilising smaller sample sizes to evaluate response to therapy. The broad array of ${ }^{129} \mathrm{Xe}$ MRI biomarkers may provide endpoints for clinical trials of novel treatments for asthma, COPD, cystic fibrosis and especially pulmonary fibrosis. The spatially resolved functional information provided by ${ }^{129} \mathrm{Xe}$ MRI may perhaps guide placement of endobronchial valves, which are currently guided using structural information from CT. Moreover, ${ }^{129} \mathrm{Xe}$ MRI may provide a way to generate quantitative pathological evidence for treatment responses, for example following bronchial thermoplasty $[45,46]$ or novel biological treatments for asthma [47], where patients experience improved quality of life and reduced exacerbation frequency, often in the absence of improvements in forced expiratory volume in $1 \mathrm{~s}$. Another valuable application of ${ }^{129} \mathrm{Xe}$ MRI may be in the prediction of pulmonary exacerbations, which has been demonstrated using ${ }^{3} \mathrm{He}$ MRI $[48,49]$. Because ${ }^{129}$ Xe MRI appears to be more sensitive to ventilation abnormalities $[23,26]$, it is expected to also predict or explain pulmonary exacerbations of COPD, cystic fibrosis and asthma.

It remains difficult to predict response to treatment in many respiratory diseases, which is becoming increasingly important as novel, expensive therapies continue to be developed. Imaging phenotypes are widely recognised in COPD and pulmonary fibrosis, and the combination of available ${ }^{129} \mathrm{Xe}$ MRI biomarkers may provide novel phenotypes to support regulatory and treatment decisions. Moreover, ${ }^{129} \mathrm{Xe}$ MRI provides a regional map of lung structure-function that can be likened to a fingerprint or "lung-print", to support individualised therapy decisions or $n=1$ studies in individual patients.

${ }^{129} \mathrm{Xe}$ MRI is particularly invaluable for longitudinal monitoring of disease progression, disease phenotypes and/or treatment response (in and outside of clinical trials) because it poses no radiation burden on patients. The lack of ionising radiation is particularly needed for examinations in vulnerable populations, such as children with chronic lung disease.

In summary, ${ }^{129} \mathrm{Xe}$ MRI provides rapid, sensitive, non-invasive and simultaneous measurements of pulmonary ventilation, lung tissue microstructure as well as diffusion within the alveolus and into the alveolar tissue and red blood cells, providing new opportunities to more deeply investigate lung diseases and unravel enigmas in respiratory medicine. 


\section{References}

1 Bergin CJ, Glover GM, Pauly J. Magnetic resonance imaging of lung parenchyma. J Thorac Imaging 1993; 8: $12-17$.

2 Johnson KM, Fain SB, Schiebler ML, et al. Optimized 3D ultrashort echo time pulmonary MRI. Magn Reson Med 2013; 70: 1241-1250.

3 Couch MJ, Ball IK, Li T, et al. Inert fluorinated gas MRI: a new pulmonary imaging modality. NMR Biomed 2014; 27: 1525-1534

4 Edelman RR, Hatabu H, Tadamura E, et al. Noninvasive assessment of regional ventilation in the human lung using oxygen-enhanced magnetic resonance imaging. Nat Med 1996; 2: 1236-1239.

5 Bergin CJ, Pauly JM, Macovski A. Lung parenchyma: projection reconstruction MR imaging. Radiology 1991; 179: 777-781.

6 Bauman G, Puderbach M, Deimling M, et al. Non-contrast-enhanced perfusion and ventilation assessment of the human lung by means of fourier decomposition in proton MRI. Magn Reson Med 2009; 62: 656-664.

7 Albert MS, Cates GD, Driehuys B, et al. Biological magnetic resonance imaging using laser-polarized 129Xe. Nature 1994; 370: 199-201.

8 Shea DA, Morgan D. The helium-3 shortage: supply, demand, and options for Congress. R41419. Washington, Congressional Research Service, 2010.

9 Hersman FW, Ruset IC, Ketel S, et al. Large production system for hyperpolarized 129Xe for human lung imaging studies. Acad Radiol 2008; 15: 683-692.

10 Nikolaou P, Coffey AM, Walkup LL, et al. Near-unity nuclear polarization with an open-source 129Xe hyperpolarizer for NMR and MRI. Proc Natl Acad Sci USA 2013; 110: 14150-14155.

11 Norquay G, Parnell SR, Xu X, et al. Optimized production of hyperpolarized 129Xe at 2 bars for in vivo lung magnetic resonance imaging. J Appl Phys 2013; 113: 044908.

12 Walker TG, Happer W. Spin-exchange optical pumping of noble-gas nuclei. Rev Mod Phys 1997; 69: 629.

13 He M, Robertson SH, Kaushik SS, et al. Dose and pulse sequence considerations for hyperpolarized (129)Xe ventilation MRI. Magn Reson Imaging 2015; 33: 877-885.

14 He M, Driehuys B, Que LG, et al. Using hyperpolarized 129Xe MRI to quantify the pulmonary ventilation distribution. Acad Radiol 2016; 23: 1521-1531.

15 Horn FC, Rao M, Stewart NJ, et al. Multiple breath washout of hyperpolarized (129) Xe and (3) He in human lungs with three-dimensional balanced steady-state free-precession imaging. Magn Reson Med 2017; 77: 2288-2295.

16 Kaushik SS, Cleveland ZI, Cofer GP, et al. Diffusion-weighted hyperpolarized 129Xe MRI in healthy volunteers and subjects with chronic obstructive pulmonary disease. Magn Reson Med 2011; 65: 1154-1165.

17 Qing K, Ruppert K, Jiang Y, et al. Regional mapping of gas uptake by blood and tissue in the human lung using hyperpolarized xenon-129 MRI. J Magn Reson Imaging 2014; 39: 346-359.

18 Kaushik SS, Robertson SH, Freeman MS, et al. Single-breath clinical imaging of hyperpolarized (129)Xe in the airspaces, barrier, and red blood cells using an interleaved 3D radial 1-point Dixon acquisition. Magn Reson Med 2016; 75: 1434-1443.

19 Mugler JP, Driehuys B, Brookeman JR, et al. MR imaging and spectroscopy using hyperpolarized 129Xe gas: preliminary human results. Magn Reson Med 1997; 37: 809-815.

20 Norquay G, Leung G, Stewart NJ, et al. (129) Xe chemical shift in human blood and pulmonary blood oxygenation measurement in humans using hyperpolarized (129) Xe MRI. Magn Reson Med 2017; 77: 1399-1408.

21 Shukla Y, Wheatley A, Kirby M, et al. Hyperpolarized 129Xe magnetic resonance imaging: tolerability in healthy volunteers and subjects with pulmonary disease. Acad Radiol 2012; 19: 941-951.

22 Driehuys B, Martinez-Jimenez S, Cleveland ZI, et al. Chronic obstructive pulmonary disease: safety and tolerability of hyperpolarized 129Xe MR imaging in healthy volunteers and patients. Radiology 2012; 262: 279-289.

23 Svenningsen S, Kirby M, Starr D, et al. Hyperpolarized (3) He and (129) Xe MRI: differences in asthma before bronchodilation. J Magn Reson Imaging 2013; 38: 1521-1530.

24 Qing K, Mugler JP, Altes TA, et al. Assessment of lung function in asthma and COPD using hyperpolarized $129 \mathrm{Xe}$ chemical shift saturation recovery spectroscopy and dissolved-phase MRI. NMR Biomed 2014; 27: 1490-1501.

25 Ebner L, He M, Virgincar RS, et al. Hyperpolarized 129Xenon magnetic resonance imaging to quantify regional ventilation differences in mild to moderate asthma: a prospective comparison between semiautomated ventilation defect percentage calculation and pulmonary function tests. Invest Radiol 2017; 52: 120-127.

26 Kirby M, Svenningsen S, Owrangi A, et al. Hyperpolarized 3He and 129Xe MR imaging in healthy volunteers and patients with chronic obstructive pulmonary disease. Radiology 2012; 265: 600-610.

27 Virgincar RS, Cleveland ZI, Kaushik SS, et al. Quantitative analysis of hyperpolarized 129Xe ventilation imaging in healthy volunteers and subjects with chronic obstructive pulmonary disease. NMR Biomed 2013; 26: 424-435.

28 Matin TN, Rahman N, Nickol AH, et al. Chronic obstructive pulmonary disease: lobar analysis with hyperpolarized (129)Xe MR imaging. Radiology 2017; 282: 857-868.

29 Ruppert K, Qing K, Patrie JT, et al. Using hyperpolarized xenon-129 MRI to quantify early-stage lung disease in smokers. Acad Radiol 2019; 26: 355-366.

30 Kanhere N, Couch MJ, Kowalik K, et al. Correlation of lung clearance index with hyperpolarized (129)Xe magnetic resonance imaging in pediatric subjects with cystic fibrosis. Am J Respir Crit Care Med 2017; 196: 1073-1075.

31 Couch MJ, Thomen R, Kanhere N, et al. A two-center analysis of hyperpolarized (129)Xe lung MRI in stable pediatric cystic fibrosis: potential as a biomarker for multi-site trials. J Cyst Fibros 2019; 18: 728-733.

32 Rayment JH, Couch MJ, McDonald N, et al. Hyperpolarised (129)Xe magnetic resonance imaging to monitor treatment response in children with cystic fibrosis. Eur Respir J 2019; 53: 1802188.

33 Dahhan T, Kaushik SS, He M, et al. Abnormalities in hyperpolarized (129)Xe magnetic resonance imaging and spectroscopy in two patients with pulmonary vascular disease. Pulm Circ 2016; 6: 126-131.

34 Wang JM, Robertson SH, Wang Z, et al. Using hyperpolarized (129)Xe MRI to quantify regional gas transfer in idiopathic pulmonary fibrosis. Thorax 2018; 73: 21-28.

35 Weatherley ND, Stewart NJ, Chan HF, et al. Hyperpolarised xenon magnetic resonance spectroscopy for the longitudinal assessment of changes in gas diffusion in IPF. Thorax 2019; 74: 500-502. 
Tahir BA, Hughes PJC, Robinson SD, et al. Spatial comparison of CT-based surrogates of lung ventilation with hyperpolarized helium-3 and xenon-129 gas MRI in patients undergoing radiation therapy. Int J Radiat Oncol Biol Phys 2018; 102: 1276-1286.

37 Walkup LL, Roach DJ, Hall CS, et al. Cyst ventilation heterogeneity and alveolar airspace dilation as early disease markers in lymphangioleiomyomatosis. Ann Am Thorac Soc 2019; 16: 1008-1016.

38 Kirby M, Heydarian M, Svenningsen $\mathrm{S}$, et al. Hyperpolarized $3 \mathrm{He}$ magnetic resonance functional imaging semiautomated segmentation. Acad Radiol 2012; 19: 141-152.

39 Sukstanskii AL, Yablonskiy DA. Lung morphometry with hyperpolarized 129Xe: theoretical background. Magn Reson Med 2012; 67: 856-866.

40 Thomen RP, Quirk JD, Roach D, et al. Direct comparison of (129) Xe diffusion measurements with quantitative histology in human lungs. Magn Reson Med 2017; 77: 265-272.

41 Kaushik SS, Freeman MS, Cleveland ZI, et al. Probing the regional distribution of pulmonary gas exchange through single-breath gas- and dissolved-phase 129Xe MR imaging. J Appl Physiol 2013; 115: 850-860.

42 Chang YV. MOXE: a model of gas exchange for hyperpolarized 129Xe magnetic resonance of the lung. Magn Reson Med 2013; 69: 884-890.

43 Stewart NJ, Leung G, Norquay G, et al. Experimental validation of the hyperpolarized (129) Xe chemical shift saturation recovery technique in healthy volunteers and subjects with interstitial lung disease. Magn Reson Med 2015; 74: 196-207.

44 Stewart NJ, Chan HF, Hughes PJC, et al. Comparison of (3) He and (129) Xe MRI for evaluation of lung microstructure and ventilation at 1.5 T. J Magn Reson Imaging 2018; 48: 632-642.

45 Castro M, Rubin AS, Laviolette M, et al. Effectiveness and safety of bronchial thermoplasty in the treatment of severe asthma: a multicenter, randomized, double-blind, sham-controlled clinical trial. Am J Respir Crit Care Med 2010; 181: 116-124.

46 Chupp G, Laviolette M, Cohn L, et al. Long-term outcomes of bronchial thermoplasty in subjects with severe asthma: a comparison of 3-year follow-up results from two prospective multicentre studies. Eur Respir J 2017; 50: 1700017.

47 McGregor MC, Krings JG, Nair P, et al. Role of biologics in asthma. Am J Respir Crit Care Med 2019; 199: 433-445.

48 Kirby M, Pike D, Coxson HO, et al. Hyperpolarized (3)He ventilation defects used to predict pulmonary exacerbations in mild to moderate chronic obstructive pulmonary disease. Radiology 2014; 273: 887-896.

49 Mummy DG, Kruger SJ, Zha W, et al. Ventilation defect percent in helium-3 magnetic resonance imaging as a biomarker of severe outcomes in asthma. J Allergy Clin Immunol 2018; 141: 1140-1141. el144. 